\title{
Influences of biotin deficiency and dietary trans-fatty acids on tissue lipids in chickens
}

\author{
BY BRUCE A. WATKINS \\ Department of Poultry Science, Virginia Polytechnic Institute and State University, \\ Blacksburg, VA 24061, USA
}

(Received 16 December 1987 - Accepted 5 September 1988)

1. The combined effects of feeding hydrogenated fats and varying the levels of biotin and linoleate $(18: 2 \omega 6)$ on polyunsaturated fatty acids were studied in the chicken.

2. Biotin deficiency signs were not exacerbated by feeding hydrogenated fats or by diets low in linoleate for $21 \mathrm{~d}$.

3. Biotin deficiency resulted in proportionately higher levels of $18: 2 \omega 6$ and $\gamma$-linolenate $(18: 3 \omega 6)$ in liver triglycerides, and lower levels of dihomo- $\gamma$-linolenate $(20: 3 \omega 6)$ in liver and heart phospholipids irrespective of the $18: 2 \omega 6$ level in the diet.

4. Biotin deficiency did not alter arachidonate $(20: 4 \omega 6)$ levels in tissue lipids at $21 \mathrm{~d}$.

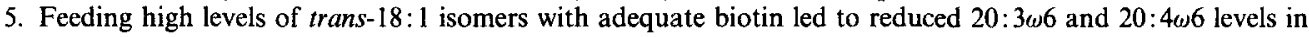
liver and heart phospholipids with compensatory increases in $\omega 3$ fatty acids.

6. The trans-isomers of 18:1 were incorporated into several tissues of the chick. Incorporation was dependent on the levels fed. Very small amounts were incorporated into brain compared with other tissues when dietary trans-isomer levels were high, but were similar when dietary trans-isomer levels were low. The trans-18:1 isomers appear to be preferentially incorporated into phospholipids as opposed to triglycerides in heart and liver.

Suboptimal biotin nutriture coupled with other nutritional and environmental stresses has been implicated in fatty liver and kidney syndrome (FLKS) which can cause high mortality in commercial flocks of meat-type chickens (Hood et al. 1976). The symptoms of FLKS differ from classical biotin deficiency in that the onset is sudden and death is rapid (within $24 \mathrm{~h}$ ), but this condition is responsive to biotin supplementation (Bannister, 1976). Bannister et al. (1983) suggested that FLKS results from feeding biotin-deficient, lowprotein rations. Classical biotin deficiency symptoms arise when protein levels are adequate.

A second condition involving acute death (sudden death syndrome, SDS) in chickens is thought by some (Hulan et al. 1980), but not by others (Steele et al. 1982), to be related to biotin status. SDS or 'flip-over' is characterized by unexpected cardiac arrest in fastgrowing, apparently healthy chickens (primarily male) of about 3 weeks of age. The most pronounced pathological changes found in these chickens are pulmonary congestion and vascular lesions (Ononiwu et al. 1979).

Because of biotin's role in the formation of malonyl-CoA, which is essential for de novo fatty acid synthesis and elongation of long-chain polyunsaturated fatty acids, certain alterations in tissue fatty acid composition are seen during biotin deficiency. Watkins \&

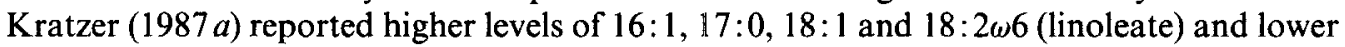
levels of $20: 3 \omega 6$ (dihomo- $\gamma$-linolenate) in the liver of biotin-deficient chicks compared with biotin-adequate counterparts. These changes have also been reported in livers of rats fed on biotin-deficient diets (Puddu et al. 1967; Kramer et al. 1984).

A critical step in essential fatty acid metabolism involving biotin may be the conversion of $\gamma$-linolenate to dihomo- $\gamma$-linolenate. A depression in dihomo- $\gamma$-linolenate results during biotin deficiency and this may lead to alterations in prostaglandin biosynthesis which could interfere with the regulation of cardiac function. Watkins \& Kratzer (1987b) reported decreased levels of prostaglandin $\mathrm{E}_{2}$ in heart tissue of biotin-deficient chicks. 
Giving certain dietary lipids may complicate the effects of biotin deficiency and contribute to the development of FLKS and SDS. Today's poultry diets often contain spent restaurant greases and hydrogenated fats. Prolonged heating and catalytic hydrogenation result in the formation of positional and geometrical isomers of unsaturated fatty acids, particularly of $18: 1$. Studies with rats have shown that some of these isomers inhibit the $\Delta 6$ - and $\Delta 5$-desaturase enzymes which are necessary for the conversion of linoleate to arachidonate (Mahfouz et al. 1980; Hill et al. 1982; Lawson et al. 1985). In addition, hydrogenated fats often contain low levels of linoleate (Thomassen et al. 1984). The presence of trans-18:1 in poultry diets, and the possibility of reduced desaturation of linoleate, might further jeopardize the synthesis of prostaglandins by reducing the substrate.

The present study was designed to examine the combined effects of biotin deficiency and the feeding of diets containing hydrogenated fats or low levels of linoleate to young chickens. The primary objectives were: (1) to determine whether biotin deficiency symptoms were exacerbated by feeding hydrogenated fats or low linoleate; (2) to measure the incorporation of geometrical isomers of $18: 1$ into chick tissues; (3) to determine the effects of the dietary treatments on fatty acid profiles in heart, liver, brain, abdominal fat pads and lungs.

\section{MATERIALS AND METHODS}

\section{Diets, animals and tissues}

Twelve experimental diets were prepared from the basal diet which was adequate in all nutrients for chickens (National Research Council, 1984) except biotin and linoleate (Table 1). Each diet contained added biotin at 0,200 or $400 \mu \mathrm{g} / \mathrm{kg}$ diet and $50 \mathrm{~g} / \mathrm{kg}$ of one of the following lipid sources: maize oil (MO); partially hydrogenated soya-bean oil (HSBO); a mixture of triolein, tripalmitin and tristearin (30:30:40, by wt; OPS); or spent restaurant grease (SRG). The purified triglycerides were purchased from the United States Biochemical Corporation (Cleveland, $\mathrm{OH}$ ). Dried egg albumen was added to the diets as a source of avidin which binds biotin. All diets contained 14.2 MJ metabolizable energy and $246 \mathrm{~g}$ crude protein (nitrogen $\times 6.25$ ) $/ \mathrm{kg}$.

Male broilers (Hubbard strain; 1-d-old) were randomly assigned to the twelve treatments with two replicate pens per treatment and eight chickens per pen. The chickens were maintained in battery brooders with a daily cycle of $18 \mathrm{~h}$ light $-6 \mathrm{~h}$ dark $(06 \cdot 00-24 \cdot 00$ hours light). Feed and water were provided $a d$ lib. for the duration of the $21 \mathrm{~d}$ experiment. Total feed consumptions and weight gains were determined at the completion of the experiment for calculation of feed efficiency (total gain/total feed) on a pen basis.

On day 21, chickens were weighed and scored for twisted leg and dermatitis on a scale of 0-4 (Watkins \& Kratzer, 1987a). Chickens were killed by cervical dislocation. Tissues were surgically removed and frozen at $-20^{\circ}$ for subsequent analyses for biotin content in liver and heart, and fatty acid compositions of liver and heart phospholipids and triglycerides. The total trans-18:1 fatty acids were measured in brain, abdominal fat pads, heart, liver and lungs and in phospholipids and triglycerides of heart and liver.

\section{Analytical procedures}

Biotin was measured by the radiochemical method of Hood (1977) in liver and heart from chicks fed on MO. The number of chick tissues assayed varied with treatment.

Total lipids from brain, fat pads, heart, liver and lungs were extracted in chloroform-methanol $(2: 1, \mathrm{v} / \mathrm{v})$ by the method of Bligh \& Dyer (1959). In addition, triglycerides (TG) and phospholipids (PL) were separated in lipid extracts from hearts and livers using thin-layer chromatography (TLC), with a developing cocktail of light 
Table 1. Composition of the basal diet $(\mathrm{g} / \mathrm{kg}$ diet $)$

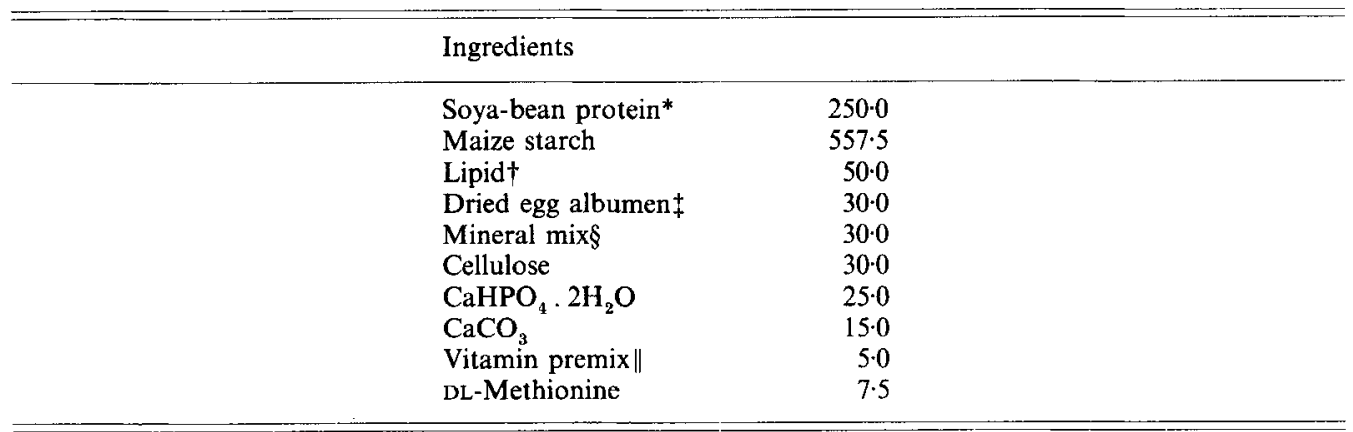

* Ralston Purina Assay Protein, RP-101, purchased from Ralston Purina Company, Richland, IN, contained the following $(\mathrm{g} / \mathrm{kg})$ : moisture 55 , crude protein (nitrogen $\times 6.25$ ) 903 , ash 38 , fat 3 , fibre 1 .

$\uparrow$ One of the following lipid sources: maize oil (MO), hydrogenated soya-bean oil (HSBO), trioleintripalmitin-tristearin mixture (30:30:40, by wt; OPS) or spent restaurant grease (SRG).

$\ddagger$ Dried egg albumen supplied by Henningsen Foods, Omaha, NE, contained the following $(\mathrm{g} / \mathrm{kg}):$ moisture 80 , protein 800 , analysed avidin binding was 5.86 units $/ \mathrm{g}$ ( 1 unit of avidin binds $1 \mu \mathrm{g}$ biotin).

\$ Contained (mg/kg diet): $\mathrm{CoCl}_{2} .6 \mathrm{H}_{2} \mathrm{O} 5, \mathrm{CuSO}_{4} .5 \mathrm{H}_{2} \mathrm{O} 60, \mathrm{FeSO}_{4} .7 \mathrm{H}_{2} \mathrm{O} 500, \mathrm{KCl} 1500, \mathrm{~K}_{2} \mathrm{HPO}_{4} 6000$, $\mathrm{KIO}_{3} 6, \mathrm{MgSO}_{4} .7 \mathrm{H}_{2} \mathrm{O} 6000, \mathrm{MnSO}_{4} . \mathrm{H}_{2} \mathrm{O} 300, \mathrm{NaCl} 6000, \mathrm{Na}_{2} \mathrm{MoO}_{4} .2 \mathrm{H}_{2} \mathrm{O} 10, \mathrm{Na}_{2} \mathrm{SeO}_{3} 0.43, \mathrm{ZnO} 90$.

$\|$ Contained $(\mathrm{mg} / \mathrm{kg}$ diet): retinol 1.35 , cholecalciferol $0 \cdot 1125$, DL- $\alpha$-tocopheryl acetate 50 , menadione $1 \cdot 5$, thiamin 15 , riboflavin 15 , calcium pantothenate 15 , niacin 50 , pyridoxine 6 , folic acid 6 , cyanocobalamin 0.02 , choline chloride 1000 , butylated hydroxytoluene 200 .

petroleum (b.p. $\left.35-60^{\circ}\right)$-diethyl ether-glacial acetic acid $(80: 20: 1$, by vol.). The fatty acids in TG and PL were methylated (Metcalfe et al. 1966) and fatty acid profiles were determined with an HP 5890A gas-liquid chromatograph equipped with a flame-ionization detector and a Nelson Analytical Data System (Hewlett Packard Co., Sunnyvale, CA). A DB225 fused silica column $(0.25 \mathrm{~mm}$ i.d. $\times 30 \mathrm{~m})$ purchased from J \& W Scientific Co. (Folsom, CA) was used with helium as the carrier gas and temperature programming. Fatty acid standards were purchased from Nu-Chek-Prep, Inc. (Elysian, MN). Proportions of fatty acids present in each sample were determined as area \%. Eight chicks per treatment were used except where insufficient tissue was available.

Fatty acid analysis was also done for each dietary lipid source, and the cis-: trans-isomer ratios of 16:1 and 18:1 in HSBO and SRG were determined in triplicate by combined argentation-TLC (Dudley \& Anderson, 1975) and gas-liquid chromatography (as described previously). Silver nitrate-impregnated TLC plates were prepared by immersing precoated plates into a saturated methanolic solution of silver nitrate. Methylated samples of each lipid were applied, and the plates were developed twice in hexane-diethyl ether-glacial acetic acid (94:4:2, by vol.). Methyl nonadecanoate was added as an internal standard to the recovered fractions which were then analysed by gas-liquid chromatography. Cis:trans-isomer ratios in tissue lipids (brain, fat pads, heart, liver, lungs, and heart and liver TG and PL) were also determined for eight chicks fed on diets containing HSBO and SRG with $400 \mu \mathrm{g}$ of added biotin $/ \mathrm{kg}$ diet.

\section{Statistical analyses}

Values for body-weights, feed efficiencies, and twisted leg and dermatitis scores were subjected to an analysis of variance (Snedecor \& Cochran, 1974) with a $4 \times 3$ factorial arrangement of treatments (four dietary lipids with three levels of biotin). Values for biotin levels in liver and heart were subjected to a one-way design of analysis of variance (Snedecor \& Cochran, 1974). Fatty acid values were evaluated as a $4 \times 2$ factorial 
Table 2. Fatty acid composition (area \%) of dietary lipids

(Values are means of four analyses)

\begin{tabular}{|c|c|c|c|c|}
\hline \multirow[b]{2}{*}{ Fatty acid } & \multicolumn{4}{|c|}{ Dietary lipid source } \\
\hline & MO & HSBO & OPS & SRG \\
\hline $14: 0$ & $3 \cdot 32$ & 0.00 & 5.47 & $4 \cdot 13$ \\
\hline $14: 1$ & $0-00$ & $0 \cdot 00$ & $0 \cdot 27$ & $0 \cdot 72$ \\
\hline $15: 0$ & 0.00 & 0.00 & $0 \cdot 10$ & 0.69 \\
\hline $16: 0$ & $12 \cdot 53$ & 11.42 & 31.55 & $28 \cdot 15$ \\
\hline$t-16: 1$ & 0.00 & $0-00$ & 0.00 & $0 \cdot 18$ \\
\hline$c-16: 1$ & 0.11 & 0.00 & 1.60 & 2.76 \\
\hline $17: 0$ & 0.08 & $0 \cdot 12$ & 0.05 & 1.60 \\
\hline $18: 0$ & $2 \cdot 34$ & $8 \cdot 04$ & $35 \cdot 10$ & 16.77 \\
\hline$t-18: 1$ & $0 \cdot 00$ & $17 \cdot 16$ & $0 \cdot 00$ & $6 \cdot 37$ \\
\hline$c-18: 1$ & $26 \cdot 37$ & 23.53 & $20 \cdot 68$ & $30 \cdot 05$ \\
\hline$t-18: 2$ & $0 \cdot 00$ & $1 \cdot 01$ & $0 \cdot 10$ & $0 \cdot 36$ \\
\hline $18: 2 \omega 6$ & $53 \cdot 29$ & $31 \cdot 67$ & $2 \cdot 18$ & $3 \cdot 64$ \\
\hline $18: 3 \omega 3$ & 0.84 & $4 \cdot 19$ & $0 \cdot 02$ & $0 \cdot 13$ \\
\hline $20: 0$ & 0.43 & $0 \cdot 31$ & $0 \cdot 25$ & $0 \cdot 14$ \\
\hline
\end{tabular}

MO, maize oil ; HSBO, hydrogenated soya-bean oil; OPS, triolein-tripalmitin-tristearin mixture $(30: 30: 40$, by wt.); SRG, spent restaurant grease; $t$, trans-isomer; $c$, cis-isomer.

Table 3. Body-weights (at 21 d), feed efficiencies (total gain/total feed intake), twisted leg and dermatitis scores of chicks fed on the experimental diets*

(Values are means for the number of chicks shown)

\begin{tabular}{|c|c|c|c|c|c|c|}
\hline & $\begin{array}{c}\text { Biotin } \\
\text { level } \\
(\mu \mathrm{g} / \mathrm{kg})\end{array}$ & $\begin{array}{c}\text { Average } \\
\text { body-wt } \\
\text { (g) }\end{array}$ & $n$ & $\begin{array}{c}\text { Feed } \\
\text { efficiency }\end{array}$ & $\begin{array}{c}\text { Twisted } \\
\text { leg. }\end{array}$ & Dermatitis \\
\hline MO & $\begin{array}{r}0 \\
200 \\
400\end{array}$ & $\begin{array}{l}245^{\mathrm{d}} \\
440^{\mathrm{b}} \\
501^{\mathrm{a}}\end{array}$ & $\begin{array}{l}16 \\
15 \\
15\end{array}$ & $\begin{array}{l}0 \cdot 58^{\mathrm{e}} \\
0 \cdot 79^{\mathrm{a}} \\
0 \cdot 77^{\mathrm{ab}}\end{array}$ & $\begin{array}{l}2 \cdot 69^{\mathrm{b}} \\
0 \cdot 53^{\mathrm{c}} \\
0 \cdot 37^{\mathrm{c}}\end{array}$ & $\begin{array}{l}3 \cdot 88^{\mathrm{a}} \\
0 \cdot 23^{\text {bcd }} \\
0.07^{\mathrm{cd}}\end{array}$ \\
\hline HSBO & $\begin{array}{r}0 \\
200 \\
400\end{array}$ & $\begin{array}{l}236^{\mathrm{d}} \\
510^{\mathrm{a}} \\
503^{\mathrm{a}}\end{array}$ & $\begin{array}{l}15 \\
16 \\
15\end{array}$ & $\begin{array}{l}0.54^{\mathrm{f}} \\
0.76^{\text {abe }} \\
0.75^{\text {abe }}\end{array}$ & $\begin{array}{l}3 \cdot 27^{\mathrm{a}} \\
0 \cdot 12^{\mathrm{c}} \\
0 \cdot 37^{\mathrm{c}}\end{array}$ & $\begin{array}{l}3.87^{\mathrm{a}} \\
0.32^{\mathrm{bed}} \\
0.07^{\mathrm{cd}}\end{array}$ \\
\hline OPS & $\begin{array}{r}0 \\
200 \\
400\end{array}$ & $\begin{array}{l}237^{\mathrm{d}} \\
385^{\mathrm{c}} \\
412^{\mathrm{bc}}\end{array}$ & $\begin{array}{l}14 \\
15 \\
14\end{array}$ & $\begin{array}{l}0 \cdot 53^{f} \\
0 \cdot 71^{\text {bed }} \\
0 \cdot 68^{d}\end{array}$ & $\begin{array}{l}2 \cdot 29^{\mathrm{b}} \\
0 \cdot 13^{\mathrm{c}} \\
0 \cdot 00^{\mathrm{c}}\end{array}$ & $\begin{array}{l}4 \cdot 00^{\mathrm{a}} \\
0 \cdot 40^{\mathrm{b}} \\
0 \cdot 07^{\mathrm{ed}}\end{array}$ \\
\hline SRG & $\begin{array}{r}0 \\
200 \\
400\end{array}$ & $\begin{array}{l}268^{\mathrm{d}} \\
400^{\mathrm{bc}} \\
439^{\mathrm{b}}\end{array}$ & $\begin{array}{l}15 \\
15 \\
13\end{array}$ & $\begin{array}{l}0 \cdot 58^{\mathrm{e}} \\
0 \cdot 75^{\text {abc }} \\
0 \cdot 70^{\text {cd }}\end{array}$ & $\begin{array}{l}2.53^{i)} \\
0 \cdot 00^{c} \\
0 \cdot 04^{c}\end{array}$ & $\begin{array}{l}3 \cdot 93^{\mathrm{a}} \\
0 \cdot 33^{\mathrm{bc}} \\
0 \cdot 00^{\mathrm{d}}\end{array}$ \\
\hline Pooled SD & & 65 & & 0.03 & $0 \cdot 76$ & $0 \cdot 39$ \\
\hline $\begin{array}{l}\text { Significant } \\
\text { effects }\end{array}$ & & $\begin{array}{c}\text { Biotin, } \\
\text { lipid }\end{array}$ & & $\begin{array}{c}\text { Biotin, } \\
\text { lipid }\end{array}$ & $\begin{array}{c}\text { Biotin, } \\
\text { lipid }\end{array}$ & Biotin \\
\hline
\end{tabular}

MO, maize oil; HSBO, hydrogenated soya-bean oil; OPS, triolein-tripalmitin-tristearin mixture $(30: 30: 40$, by wt); SRG, spent restaurant grease.

a-f Means within a column with different superscript letters were significantly different, as determined by Duncan's multiple-range test $(P<0 \cdot 01)$.

* For details, see Tables 1 and 2 . 
Table 4. Biotin levels in liver and heart of chicks fed on maize oil*

(Values are means with their standard errors for the number of chicks shown)

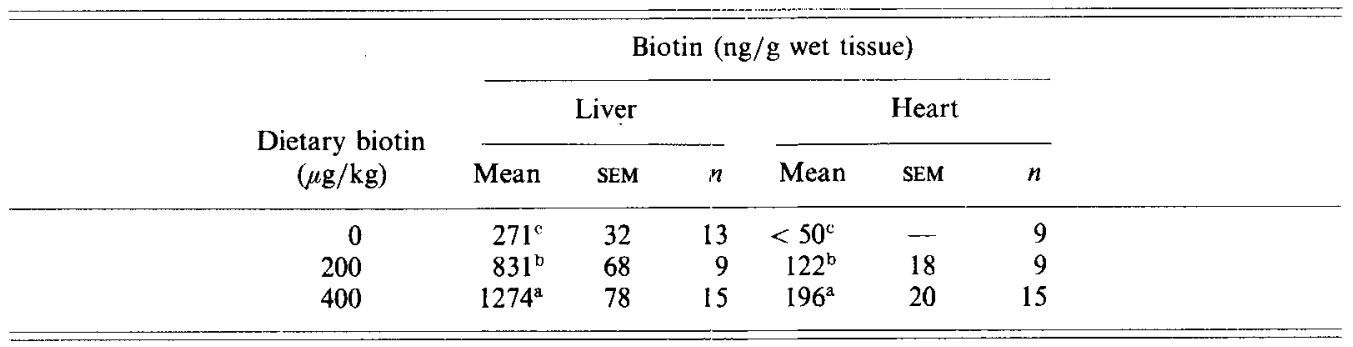

a, b, c Means within a column with different superscript letters were significantly different, as determined by Bonferroni's multiple-comparison post-priori test $(P<0.05)$.

* For details of diet, see Tables 1 and 2.

Table 5. Fatty acid composition of liver triglycerides in the chick at $21 d$ of age* (Values are means and pooled standard deviations for individual fatty acids)

\begin{tabular}{|c|c|c|c|c|c|c|c|}
\hline \multirow{2}{*}{$\begin{array}{l}\text { Fatty } \\
\text { acid } \dagger\end{array}$} & \multirow{2}{*}{$\begin{array}{c}\text { Dietary } \\
\text { biotin } \\
(\mu \mathrm{g} / \mathrm{kg})\end{array}$} & \multicolumn{4}{|c|}{ Fatty acids (area \%) } & \multirow{2}{*}{$\begin{array}{l}\text { Pooled } \\
\text { SD }\end{array}$} & \multirow{2}{*}{$\begin{array}{l}\text { Statistically } \\
\text { significant } \\
\text { effects }\end{array}$} \\
\hline & & MO & HSBO & OPS & SRG & & \\
\hline \multirow[t]{2}{*}{$16: 0$} & 0 & $27 \cdot 61^{\mathrm{c}}$ & $29 \cdot 13^{\mathrm{bc}}$ & $30.59^{\mathrm{be}}$ & $32 \cdot 86^{\mathrm{abc}}$ & $5 \cdot 02$ & Biotin \\
\hline & 400 & $38 \cdot 33^{a}$ & $35 \cdot 22^{\mathrm{ab}}$ & $37 \cdot 36^{\mathrm{a}}$ & $35 \cdot 41^{\mathrm{ab}}$ & & \\
\hline \multirow[t]{2}{*}{$16: 1$} & 0 & $8 \cdot 88^{\mathrm{ab}}$ & $6.63^{\mathrm{be}}$ & $12 \cdot 74^{\mathrm{a}}$ & $10 \cdot 39^{a b}$ & $3 \cdot 36$ & Biotin, lipid \\
\hline & 400 & $3 \cdot 61^{\mathrm{c}}$ & $6 \cdot 17^{\mathrm{bc}}$ & $6 \cdot 46^{\mathrm{bc}}$ & $4 \cdot 32^{c}$ & & \\
\hline \multirow[t]{2}{*}{$17: 0$} & 0 & $0 \cdot 24^{b}$ & $0.23^{b}$ & $0 \cdot 26^{\mathrm{b}}$ & $0 \cdot 56^{a}$ & $0 \cdot 18$ & Lipid \\
\hline & 400 & $0 \cdot 15^{b}$ & $0.29^{\mathrm{b}}$ & $0.24^{b}$ & $0 \cdot 68^{\mathrm{a}}$ & & \\
\hline \multirow[t]{2}{*}{$18: 0$} & 0 & $6 \cdot 14^{d}$ & $8 \cdot 21^{\mathrm{ed}}$ & $7.97^{\mathrm{ed}}$ & $5 \cdot 76^{\mathrm{d}}$ & $4 \cdot 37$ & Biotin \\
\hline & 400 & $17 \cdot 24^{a b}$ & $12 \cdot 71^{\mathrm{abc}}$ & $12 \cdot 00^{\mathrm{bc}}$ & $17 \cdot 85^{\mathrm{a}}$ & & \\
\hline \multirow[t]{2}{*}{$18: 1$} & 0 & $25 \cdot 20^{\mathrm{b}}$ & $29 \cdot 67^{\mathrm{ab}}$ & $34.04^{\mathrm{a}}$ & $34 \cdot 14^{a}$ & $5 \cdot 15$ & Lipid \\
\hline & 400 & $28 \cdot 55^{\mathrm{ab}}$ & $30 \cdot 28^{\mathrm{ab}}$ & $32 \cdot 10^{\mathrm{ab}}$ & $30 \cdot 23^{a b}$ & & \\
\hline \multirow[t]{2}{*}{$18: 2 \omega 6$} & 0 & $23 \cdot 28^{\mathrm{a}}$ & $16 \cdot 46^{\mathrm{b}}$ & $5 \cdot 72^{\mathrm{c}}$ & $7 \cdot 20^{\mathrm{c}}$ & $2 \cdot 15$ & $\begin{array}{l}\text { Biotin-lipid } \\
\text { interaction }\end{array}$ \\
\hline & 400 & $7 \cdot 26^{\mathrm{c}}$ & $5 \cdot 20^{\mathrm{cd}}$ & $2 \cdot 87^{\mathrm{a}}$ & $4 \cdot 74^{\mathrm{cd}}$ & & \\
\hline \multirow[t]{2}{*}{$18: 3 \omega 3$} & 0 & $0.39^{\text {bc }}$ & $0.84^{\mathrm{a}}$ & $0.46^{b}$ & $0 \cdot 19^{\mathrm{cd}}$ & $0 \cdot 19$ & $\begin{array}{l}\text { Biotin-lipid } \\
\text { interaction }\end{array}$ \\
\hline & 400 & $0 \cdot 13^{d}$ & $0 \cdot 40^{\mathrm{be}}$ & $0 \cdot 1.7^{\text {ed }}$ & $0 \cdot 24^{\text {bed }}$ & & \\
\hline \multirow[t]{2}{*}{$18: 3 \omega 6$} & 0 & $0.65^{\mathrm{a}}$ & $0 \cdot 58^{\mathrm{a}}$ & $0 \cdot 74^{a}$ & $0 \cdot 28^{\mathrm{b}}$ & 0.23 & Biotin \\
\hline & 400 & $0 \cdot 17^{\mathrm{b}}$ & $0.09^{\mathrm{b}}$ & $0 \cdot 09^{b}$ & $0.02^{\mathrm{b}}$ & & \\
\hline \multirow[t]{2}{*}{$20: 3 \omega 6$} & 0 & $0 \cdot 18$ & $0 \cdot 19$ & $0 \cdot 30$ & $0 \cdot 15$ & 0.08 & $\ldots$ \\
\hline & 400 & 0.23 & $0 \cdot 20$ & $0 \cdot 12$ & $0 \cdot 19$ & & \\
\hline \multirow{2}{*}{$20: 4 \omega 6$} & 0 & $1.91^{\mathrm{a}}$ & $1 \cdot 30^{\mathrm{ab}}$ & $1 \cdot 10^{\mathrm{gb}}$ & $1 \cdot 00^{\mathrm{ab}}$ & 0.78 & Biotin \\
\hline & 400 & $0.72^{\mathrm{b}}$ & $0.94^{\mathrm{ab}}$ & $0 \cdot 35^{b}$ & $0.80^{b}$ & & \\
\hline
\end{tabular}

MO, maize oil; HSBO, hydrogenated soya-bean oil; OPS, triolein-tripalmitin-tristearin mixture $(30: 30: 40$, by wt); SRG, spent restaurant grease.

${ }_{a}-\mathrm{d}$ For individual fatty acids, values not sharing a common superscript letter were significantly different $(P<$ $0 \cdot 05)$.

* Total chicks sampled for 0 and $400 \mu$ g biotin respectively, MO, $n$ 5, 8; HSBO, $n 4$, 6; OPS, $n$ 6, 5; SRG, $n$ 6, 7. Details of extraction and separation are described on pp. 100-101.

$\uparrow$ Only the major fatty acids are listed. 
Table 6. Fatty acid composition of heart triglycerides in the chick at $21 d$ of age* (Values are means and pooled standard deviations for individual fatty acids)

\begin{tabular}{|c|c|c|c|c|c|c|c|}
\hline \multirow{2}{*}{$\begin{array}{l}\text { Fatty } \\
\text { acid } \uparrow\end{array}$} & \multirow{2}{*}{$\begin{array}{c}\text { Dietary } \\
\text { biotin } \\
(\mu \mathrm{g} / \mathrm{kg})\end{array}$} & \multicolumn{4}{|c|}{ Fatty acids (area \%) } & \multirow{2}{*}{$\begin{array}{c}\text { Pooled } \\
\text { SD }\end{array}$} & \multirow{2}{*}{$\begin{array}{c}\text { Statistically } \\
\text { significant } \\
\text { effects }\end{array}$} \\
\hline & & MO & HSBO & OPS & SRG & & \\
\hline \multirow[t]{2}{*}{$16: 0$} & 0 & $23 \cdot 81^{b}$ & $25 \cdot 34^{\mathrm{ab}}$ & $25 \cdot 81^{\mathrm{ab}}$ & $26 \cdot 20^{\mathrm{ab}}$ & $2 \cdot 46$ & Lipid \\
\hline & 400 & $24 \cdot 57^{\mathrm{ab}}$ & $24 \cdot 84^{\mathrm{ab}}$ & $27 \cdot 43^{\mathrm{s}}$ & $26 \cdot 77^{\mathrm{ab}}$ & & \\
\hline \multirow[t]{2}{*}{$16: 1$} & 0 & $4 \cdot 60^{\mathrm{b}}$ & $5 \cdot 06^{b}$ & $9 \cdot 04^{\mathrm{a}}$ & $7 \cdot 14^{\mathrm{ab}}$ & $2 \cdot 63$ & Lipid \\
\hline & 400 & $4 \cdot 61^{\mathrm{b}}$ & $5 \cdot 34^{\mathrm{b}}$ & $7 \cdot 47^{a b}$ & $6 \cdot 26^{\mathrm{ab}}$ & & \\
\hline \multirow[t]{2}{*}{$17: 0$} & 0 & $0 \cdot 80^{\mathrm{a}}$ & $0.59^{a b}$ & $0.40^{\text {ned }}$ & $0.57^{\mathrm{abc}}$ & 0.23 & Biotin, lipid \\
\hline & 400 & $0 \cdot 23^{\mathrm{d}}$ & $0.29^{\mathrm{ed}}$ & $0 \cdot 19^{\circ}$ & $0 \cdot 56^{\mathrm{abc}}$ & & \\
\hline \multirow[t]{2}{*}{$18: 0$} & 0 & 17.09 & $15 \cdot 94$ & $10 \cdot 86$ & 9.09 & $5 \cdot 43$ & - \\
\hline & 400 & $10 \cdot 27$ & $10 \cdot 77$ & $9 \cdot 96$ & 11.52 & & \\
\hline \multirow[t]{2}{*}{$18: 1$} & 0 & $21.09^{\mathrm{e}}$ & $27 \cdot 49^{d}$ & $37 \cdot 47^{a b}$ & $39 \cdot 41^{\mathrm{ab}}$ & $5 \cdot 39$ & Biotin, lipid \\
\hline & 400 & $30 \cdot 06^{\mathrm{ed}}$ & $34 \cdot 83^{\text {be }}$ & $41 \cdot 63^{a}$ & $39 \cdot 22^{\mathrm{ab}}$ & & \\
\hline \multirow[t]{2}{*}{$18: 2 \omega 6$} & 0 & $16.03^{b}$ & $14 \cdot 36^{\mathrm{b}}$ & $7 \cdot 19^{\circ}$ & $8.83^{c}$ & $2 \cdot 81$ & Lipid interaction \\
\hline & 400 & $22 \cdot 71^{\mathrm{a}}$ & $14 \cdot 44^{b}$ & $5.93^{c}$ & $6 \cdot 76^{c}$ & & \\
\hline \multirow[t]{2}{*}{$18: 3 \omega 3$} & 0 & $0.61^{b c}$ & $1 \cdot 02^{a b}$ & $0.37^{c}$ & $0 \cdot 36^{\mathrm{c}}$ & 0.41 & Biotin, lipid \\
\hline & 400 & $0.74^{\mathrm{bc}}$ & $1 \cdot 39^{\mathrm{a}}$ & $0 \cdot 42^{c}$ & $0.82^{\mathrm{bc}}$ & & \\
\hline \multirow[t]{2}{*}{$18: 3 \omega 6$} & 0 & $0 \cdot 12$ & 0.13 & 0.12 & $0 \cdot 10$ & 0.08 & - \\
\hline & 400 & $0 \cdot 19$ & $0 \cdot 10$ & $0 \cdot 07$ & 0.05 & & \\
\hline \multirow[t]{2}{*}{$20: 3 \omega 6$} & 0 & $0 \cdot 36^{\mathrm{b}}$ & $0.22^{\mathrm{b}}$ & $0 \cdot 27^{\mathrm{b}}$ & $0 \cdot 30^{b}$ & $0 \cdot 14$ & Lipid \\
\hline & 400 & $0.57^{\mathrm{a}}$ & $0 \cdot 34^{\mathrm{b}}$ & $0 \cdot 24^{b}$ & $0 \cdot 20^{\mathrm{b}}$ & & \\
\hline \multirow[t]{2}{*}{$20: 4 * 6$} & 0 & $1 \cdot 88^{a}$ & $1 \cdot 31^{\mathrm{ab}}$ & $0.43^{\mathrm{bc}}$ & $0.67^{\text {be }}$ & 0.69 & Biotin, lipid \\
\hline & 400 & $0.91^{b c}$ & $0.73^{\text {be }}$ & $0.43^{\mathrm{be}}$ & $0 \cdot 27^{c}$ & & \\
\hline
\end{tabular}

MO, maize oil; HSBO, hydrogenated soya-bean oil; OPS, triolein-tripalmitin-tristearin mixture (30:30:40, by wt); SRG, spent restaurant grease.

${ }_{a-e}$ For individual fatty acids, values not sharing a common superscript letter were significantly different $(P<$ 0.05).

* Total chicks samples for 0 and $400 \mu$ g biotin respectively, MO, $n 5,8$; HSBO, $n 7,8$; OPS, $n 5$, 6; SRG, $n 6$, 7. Details of extraction and separation are described on pp. 100-101.

$\dagger$ Only the major fatty acids are listed.

arrangement of treatments (four dietary lipids with two levels of biotin). The statistical models did consider variation between chicks within pens and between pens for all values except feed efficiences. Bonnferroni's multiple-comparison post-priori test or Duncan's multiple-range test were employed when significant differences existed (Neter \& Wasserman, 1974). The standard error of the mean was calculated for liver biotin levels. Variation between means for performance values, deficiency symptoms and fatty acid values was expressed as a pooled standard deviation (the square root of the mean square for error) since sample sizes were unequal. Data were presented to indicate individual treatment means and the significant main effects and interactions. The statistical models used revealed no significant pen effect on the measurements taken.

\section{RESULTS}

A fatty acid compositional analysis of the four dietary lipid sources is given in Table 2 . The MO diets contained high levels of linoleate and no trans-isomers of 18:1. The diets containing HSBO had adequate amounts of linoleate $(15.8 \mathrm{~g} / \mathrm{kg}$ diet $)$ and $42 \%$ of the $18: 1$ fatty acids were trans-isomers. Diets containing SRG had limiting amounts of linoleate $(1.8 \mathrm{~g} / \mathrm{kg}$ diet $)$ and $17 \%$ of the $18: 1$ fatty acids were trans-isomers. When diets contained OPS, no unnatural positional or geometrical isomers of 18:1 were present and linoleate 
Table 7. Fatty acid composition of liver phospholipids in the chick at $21 \mathrm{~d}$ of age* (Values are means and pooled standard deviations for individual fatty acids)

\begin{tabular}{|c|c|c|c|c|c|c|c|}
\hline \multirow{2}{*}{$\begin{array}{l}\text { Fatty } \\
\text { acid } \dagger\end{array}$} & \multirow{2}{*}{$\begin{array}{c}\text { Dietary } \\
\text { biotin } \\
(\mu \mathrm{g} / \mathrm{kg})\end{array}$} & \multicolumn{4}{|c|}{ Fatty acids (area \%) } & \multirow{2}{*}{$\begin{array}{c}\text { Pooled } \\
\text { SD }\end{array}$} & \multirow{2}{*}{$\begin{array}{c}\text { Statistically } \\
\text { significant } \\
\text { effects }\end{array}$} \\
\hline & & MO & $\mathrm{HSBO}$ & OPS & SRG & & \\
\hline \multirow[t]{2}{*}{$16: 0$} & 0 & $21 \cdot 56$ & $17 \cdot 14$ & $19 \cdot 89$ & $19 \cdot 21$ & $2 \cdot 46$ & - \\
\hline & 400 & $18 \cdot 53$ & $18 \cdot 00$ & $18 \cdot 40$ & 18.98 & & \\
\hline \multirow[t]{2}{*}{$16: 1$} & 0 & $2 \cdot 41^{b}$ & $1 \cdot 88^{\mathrm{b}}$ & $6 \cdot 58^{\mathfrak{u}}$ & $3 \cdot 63^{\mathrm{a}}$ & $2 \cdot 02$ & Biotin, lipid \\
\hline & 400 & $1 \cdot 05^{\mathrm{b}}$ & $1 \cdot 64^{\circ}$ & $2 \cdot 98^{\mathrm{ab}}$ & $1 \cdot 73^{\mathrm{b}}$ & & \\
\hline \multirow[t]{2}{*}{ 17:0 } & 0 & $0.27^{\mathrm{bc}}$ & $0 \cdot 19^{\mathrm{cd}}$ & $0 \cdot 19^{\text {ed }}$ & $0 \cdot 48^{\mathrm{a}}$ & 0.05 & Biotin, lipid \\
\hline & 400 & $0 \cdot 08^{d}$ & $0.08^{d}$ & $0 \cdot 11^{d}$ & $0 \cdot 32^{\mathrm{b}}$ & & \\
\hline \multirow[t]{2}{*}{$18: 0$} & 0 & $19 \cdot 69^{b}$ & $20 \cdot 74^{b}$ & $17 \cdot 83^{b}$ & $19 \cdot 25^{b}$ & $3 \cdot 64$ & Biotin \\
\hline & 400 & $25 \cdot 55^{a}$ & $23 \cdot 65^{a}$ & $23 \cdot 20^{\mathrm{a}}$ & $23 \cdot 83^{a}$ & & \\
\hline \multirow[t]{2}{*}{$18: 1$} & 0 & $7 \cdot 36^{\complement}$ & $7.92^{e}$ & $17 \cdot 03^{a}$ & $15 \cdot 22^{\mathrm{ub}}$ & $3 \cdot 68$ & Biotin, lipid \\
\hline & 400 & $9 \cdot 71^{\mathrm{bc}}$ & $14 \cdot 10^{\mathrm{abc}}$ & $16 \cdot 37^{\mathrm{ab}}$ & $13 \cdot 75^{\mathrm{abc}}$ & & \\
\hline \multirow[t]{2}{*}{$18: 2 \omega 6$} & 0 & $22 \cdot 48^{a}$ & $20 \cdot 93^{\mathrm{ab}}$ & $11 \cdot 92^{\mathrm{e}}$ & $14 \cdot 72^{\mathrm{d}}$ & $1 \cdot 38$ & $\begin{array}{r}\text { Biotin-lipid } \\
\text { interaction }\end{array}$ \\
\hline & 400 & $18 \cdot 81^{\mathrm{be}}$ & $17 \cdot 84^{\mathrm{c}}$ & $11.93^{\mathrm{e}}$ & $14 \cdot 48^{\text {de }}$ & & \\
\hline \multirow[t]{2}{*}{$18: 3 \omega 3$} & 0 & $0 \cdot 07^{\mathrm{b}}$ & $0 \cdot 26^{\mathrm{a}}$ & $0.21^{\mathrm{ab}}$ & $0 \cdot 15^{a b}$ & 0.08 & Lipid \\
\hline & 400 & $0 \cdot 06^{\mathrm{b}}$ & $0 \cdot 27^{\mathrm{a}}$ & $0 \cdot 14^{\mathrm{ab}}$ & $0 \cdot 17^{\text {ab }}$ & & \\
\hline \multirow[t]{2}{*}{$18: 3 \omega 6$} & 0 & $0.62^{a b}$ & $0.50^{\mathrm{at}}$ & $0.38^{\mathrm{b}}$ & $1 \cdot 00^{\mathrm{a}}$ & 0.25 & Biotin, lipid \\
\hline & 400 & $0.25^{\mathrm{b}}$ & $0 \cdot 19^{\circ}$ & $0.24^{\mathrm{b}}$ & $0 \cdot 29^{b}$ & & \\
\hline \multirow[t]{2}{*}{$20: 3 \omega 6$} & 0 & $0 \cdot 85^{\mathrm{d}}$ & $1.02^{\alpha}$ & $1 \cdot 82^{\mathrm{c}}$ & $1 \cdot 40^{\mathrm{cd}}$ & $0 \cdot 33$ & Biotin \\
\hline & 400 & $2 \cdot 63^{\mathrm{a}}$ & $2 \cdot 49^{\mathrm{ah}}$ & $2 \cdot 34^{\mathrm{ab}}$ & $2 \cdot 11^{\mathrm{ab}}$ & & \\
\hline \multirow[t]{2}{*}{$20: 4 \omega 6$} & 0 & $14 \cdot 73^{\mathrm{a}}$ & $15 \cdot 30^{a}$ & $10 \cdot 66^{\mathrm{ab}}$ & $12 \cdot 24^{\mathrm{ab}}$ & 2.73 & Biotin-lipid \\
\hline & 400 & $14 \cdot 62^{\mathrm{a}}$ & $9.53^{b}$ & $9 \cdot 72^{b}$ & $11 \cdot 62^{\mathrm{ab}}$ & & \\
\hline \multirow[t]{2}{*}{$20: 5 \omega 3$} & 0 & $0 \cdot 10^{\mathrm{e}}$ & $0.53^{\mathrm{b}}$ & $0 \cdot 62^{\mathrm{ab}}$ & $0 \cdot 60^{\mathrm{ab}}$ & $0 \cdot 15$ & Biotin, lipid \\
\hline & 400 & $0 \cdot 14^{c}$ & $0.87^{a}$ & $0.72^{a b}$ & $0 \cdot 81^{\text {ab }}$ & & \\
\hline
\end{tabular}

MO, maize oil ; HSBO, hydrogenated soya-bean oil ; OPS, triolein-tripalmitin-tristearin mixture $(30: 30: 40$, by wt); SRG, spent restaurant grease.

${ }_{a-e}$ For individual fatty acids, values not sharing a common superscript letter were significantly different $(P<$ $0 \cdot 05)$.

* Total chicks sampled for 0 and $400 \mu \mathrm{g}$ biotin respectively, MO, $n$ 5, 8 ; HSBO, $n 4,6$; OPS, $n$ 6, 5; SRG, $n$

6, 7. Details of extraction and separation are described on pp. 100-101.

$\dagger$ Only the major fatty acids are listed.

was only $1 \mathrm{~g} / \mathrm{kg}$ diet. The linoleate requirement for the chick has been established at $10 \mathrm{~g} /$ $\mathrm{kg}$ diet (National Research Council. 1984).

Body-weight, feed efficiency, and twisted leg and dermatitis values are presented in Table 3. Average body-weights were dependent on the level of biotin and the lipid source fed. There were no differences among body-weights of chicks given $0 \mu \mathrm{g}$ added biotin, indicating no confounding effects of fat source on growth depression caused by biotin deficiency. Chicks fed on $400 \mu \mathrm{g}$ added biotin and OPS or SRG weighed less than those fed on diets containing the same amount of added biotin with MO or HSBO. Chicks fed on HSBO grew most rapidly. The incidence of twisted leg was dependent on the biotin level and lipid source fed, while the dermatitis response was due only to biotin deficiency. Feed efficiency improved with the addition of biotin at $200 \mu \mathrm{g} / \mathrm{kg}$ diet for all lipid sources fed.

Biotin levels in liver and heart tissues were dependent on the amount of biotin fed (Table 4). Chicks receiving $0 \mu \mathrm{g}$ biotin had the lowest levels of this vitamin in both tissues. Levels of biotin in heart were much lower than those in liver.

Fatty acid compositions of liver TG are shown in Table 5 (only major fatty acids are 
Table 8. Fatty acid composition of heart phospholipids in the chick at $21 d$ of age* (Values are means and pooled standard deviations for individual fatty acids)

\begin{tabular}{|c|c|c|c|c|c|c|c|}
\hline \multirow{2}{*}{$\begin{array}{l}\text { Fatty } \\
\text { acidt }\end{array}$} & \multirow{2}{*}{$\begin{array}{c}\text { Dietary } \\
\text { biotin } \\
(\mu \mathrm{g} / \mathrm{kg})\end{array}$} & \multicolumn{4}{|c|}{ Fatty acid (area \%) } & \multirow{2}{*}{$\begin{array}{c}\text { Pooled } \\
\text { SD }\end{array}$} & \multirow{2}{*}{$\begin{array}{l}\text { Statistically } \\
\text { significant } \\
\text { effects }\end{array}$} \\
\hline & & MO & HSBO & OPS & SRG & & \\
\hline \multirow[t]{2}{*}{$16: 0$} & 0 & $14 \cdot 48$ & $15 \cdot 85$ & 14.95 & $14 \cdot 54$ & 1.56 & - \\
\hline & 400 & $15 \cdot 71$ & $14 \cdot 72$ & 16.49 & $14 \cdot 66$ & & \\
\hline \multirow[t]{2}{*}{$16: 1$} & 0 & $0.72^{\mathrm{bc}}$ & $1 \cdot 19^{a b c}$ & $1 \cdot 84^{\mathrm{u}}$ & $1.53^{a b}$ & 0.51 & Biotin, lipid \\
\hline & 400 & $0 \cdot 47^{\circ}$ & $0.72^{\text {be }}$ & $1 \cdot 44^{\mathrm{abc}}$ & $1 \cdot 07^{a b c}$ & & \\
\hline \multirow[t]{2}{*}{$17: 0$} & 0 & $0 \cdot 19^{\circ}$ & $0 \cdot 24^{\mathrm{be}}$ & $0 \cdot 20^{\mathrm{be}}$ & $0 \cdot 42^{\mathrm{a}}$ & 0.05 & Biotin, lipid \\
\hline & 400 & $0.12^{c}$ & $0.14^{\mathrm{e}}$ & $0.13^{\mathrm{c}}$ & $0.32^{\text {ab }}$ & & \\
\hline \multirow[t]{2}{*}{$18: 0$} & 0 & $16 \cdot 33^{\mathrm{b}}$ & $19 \cdot 65^{a b}$ & $21 \cdot 51^{\mathrm{ab}}$ & $20 \cdot 12^{\mathrm{ab}}$ & $3 \cdot 13$ & - \\
\hline & 400 & $22 \cdot 75^{\mathrm{a}}$ & $20.77^{\mathrm{ab}}$ & $20 \cdot 13^{\mathrm{ab}}$ & $20 \cdot 98^{a b}$ & & \\
\hline \multirow[t]{2}{*}{$18: 1$} & 0 & $7 \cdot 19^{\mathrm{c}}$ & $13.03^{\mathrm{b}}$ & $14 \cdot 13^{b}$ & $13 \cdot 74^{\mathrm{b}}$ & $1 \cdot 36$ & Biotin, lipid \\
\hline & 400 & $9.06^{\mathrm{c}}$ & $13 \cdot 40^{b}$ & $17 \cdot 56^{\mathrm{a}}$ & $15 \cdot 57^{\mathrm{ah}}$ & & \\
\hline \multirow[t]{2}{*}{$18: 2 \omega 6$} & 0 & $24 \cdot 81^{\mathrm{Bb}}$ & $23 \cdot 49^{\mathrm{ab}}$ & $22 \cdot 97^{\mathrm{ab}}$ & $25 \cdot 53^{a b}$ & $2 \cdot 24$ & Lipid \\
\hline & 400 & $24 \cdot 91^{\mathrm{ab}}$ & $26 \cdot 68^{a}$ & $22 \cdot 09^{b}$ & $22 \cdot 86^{\mathrm{ab}}$ & & \\
\hline \multirow[t]{2}{*}{$18: 3 \omega 3$} & 0 & $0-07$ & $0 \cdot 19$ & $0 \cdot 12$ & $0 \cdot 11$ & 0.12 & - \\
\hline & 400 & 0.07 & 0.19 & $0 \cdot 14$ & 0.23 & & \\
\hline \multirow[t]{2}{*}{$18: 3 \omega 6$} & 0 & $0.06^{\text {be }}$ & $0.08^{a b c}$ & $0.08^{a b c}$ & $0 \cdot 13^{\mathrm{a}}$ & 0.03 & $\begin{array}{l}\text { Biotin-lipid } \\
\text { interaction }\end{array}$ \\
\hline & 400 & $0.01^{\circ}$ & $0.06^{\mathrm{bc}}$ & $0 \cdot 11^{\mathrm{ab}}$ & $0.07^{\text {abc }}$ & & \\
\hline \multirow[t]{2}{*}{$20: 3 \omega 6$} & 0 & $0.57^{\circ}$ & $0.76^{\text {ie }}$ & $1 \cdot 43^{\mathrm{bc}}$ & $1 \cdot 34^{\mathrm{c}}$ & 0.24 & Biotin, lipid \\
\hline & 400 & $1 \cdot 2 \mathrm{I}^{\mathrm{cd}}$ & $1 \cdot 49^{\mathrm{bc}}$ & $1.93^{\mathrm{a}}$ & $1 \cdot 75^{\mathrm{ab}}$ & & \\
\hline \multirow[t]{2}{*}{$20: 4 w 6$} & 0 & $21 \cdot 03^{\mathrm{a}}$ & $16 \cdot 09^{\mathrm{at}}$ & $12 \cdot 65^{\mathrm{ed}}$ & $12 \cdot 85^{\mathrm{cd}}$ & $2 \cdot 64$ & Biotin, lipid \\
\hline & 400 & $15 \cdot 87^{\mathrm{ab}}$ & $12 \cdot 76^{\mathrm{cd}}$ & $7.95^{\mathrm{d}}$ & $10 \cdot 63^{c}$ & & \\
\hline \multirow[t]{2}{*}{$20: 5 \omega 3$} & 0 & $0 \cdot 06^{c}$ & $0.34^{\mathrm{b}}$ & $0 \cdot 42^{\mathrm{ah}}$ & $0.50^{\mathrm{ab}}$ & $0 \cdot 10$ & Biotin, lipid \\
\hline & 400 & $0 \cdot 06^{\mathrm{c}}$ & $0.49^{a b}$ & $0.56^{\mathrm{a}}$ & $0 \cdot 60^{\mathrm{a}}$ & & \\
\hline
\end{tabular}

MO, maize oil; HSBO, hydrogenated soya-bean oil; OPS, triolein-tripalmitin-tristearin mixture $(30: 30: 40$, by wt); SRG, spent restaurant grease.

a-e For individual fatty acids, values not sharing a common superscript letter were significantly different $(P<$ $0 \cdot 05)$.

* Total chicks sampled for 0 and $400 \mu \mathrm{g}$ biotin respectively, MO, $n 5,8$; HSBO, $n 7,8$; OPS, $n 5,6 ; \mathrm{SRG}, n 6$,

7. Details of extraction and separation are described on pp. 100-101.

+ Only the major fatty acids are listed.

presented so the totals are not $100 \%$ ). Chicks receiving $0 \mu \mathrm{g}$ added biotin and adequate linoleate (MO) had lower levels of 16:0 and 18:0, but higher levels of 16:1, 18:2 $\omega 6$, 18:3 $\omega 3,18: 3 \omega 6$ and $20: 4 \omega 6$ (Table 5). Biotin-deficient chicks fed on HSBO and OPS also had higher levels of $18: 2 \omega 6,18: 3 \omega 3$ and $18: 3 \omega 6$ compared with chicks receiving $400 \mu \mathrm{g}$ biotin. Higher levels of linoleate, $\alpha$-linolenate $(18: 3 \omega 3)$ and $18: 3 \omega 6$ occurred during biotin deficiency independent of the linoleate levels fed. The changes in fatty acids observed in chicks fed on SRG were not great enough to be significant.

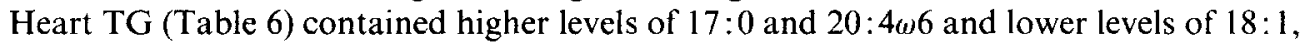
$18: 2 \omega 6$ and $20: 3 \omega 6$ in biotin-deficient chicks fed on MO compared with chicks receiving $400 \mu \mathrm{g}$ biotin. Chicks fed on OPS and SRG tended to have higher levels of 16:1 and 18:1 in liver and heart TG compared with chicks fed on MO and HSBO. This may be due to the higher levels of 16:0 and 18:0 in OPS and SRG (Table 2) and to the chick's ability to desaturase these fatty acids.

Fatty acid values in liver and heart PL are presented in Tables 7 and 8 respectively (only major fatty acids are presented so the totals are not $100 \%$ ). Observations in liver PL due to biotin deficiency included increased values for $18: 2 \omega 6$ in the MO and HSBO treatments but values for 18:0 and 20:3 w6 were decreased in all lipid treatments. Also with biotin 


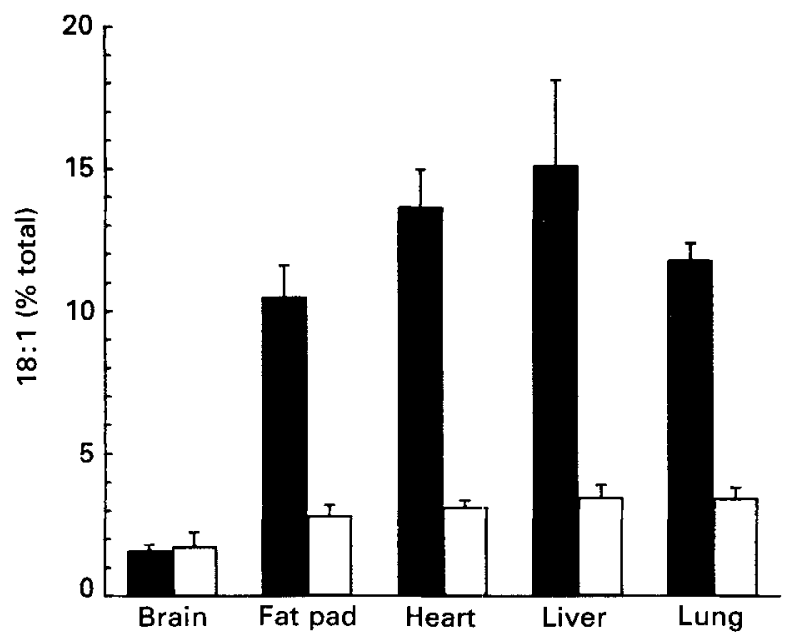

Fig. 1 Incorporation of trans-18:1 fatty acids into tissues of chicks receiving $400 \mu \mathrm{g}$ added biotin $/ \mathrm{kg}$ diet. Values are means with their standard errors represented by vertical bars for eight chicks fed on hydrogenated soya-bean oil $(\square)$ or spent restaurant grease ( $\square$ ).

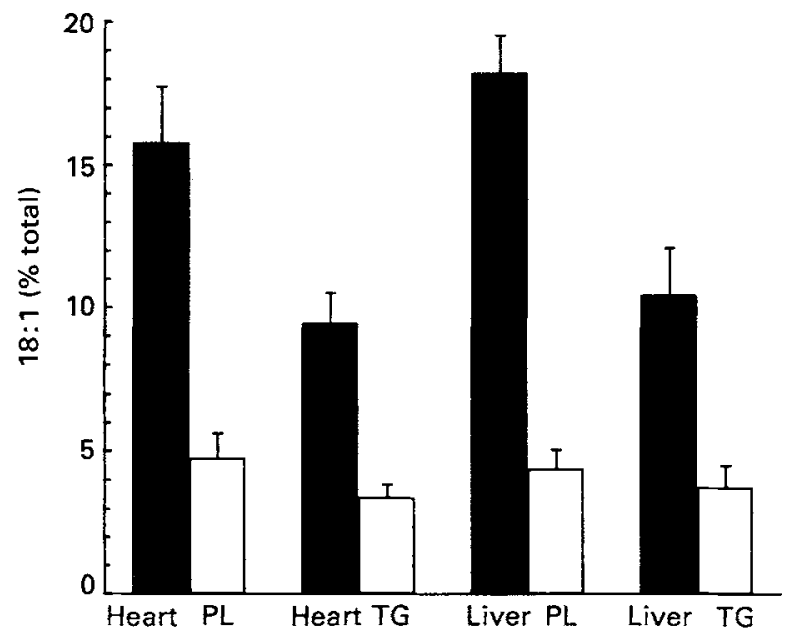

Fig. 2. Distribution of trans-18:1 fatty acids in heart and liver phospholipids (PL) and triglycerides (TG) of chicks receiving $400 \mu \mathrm{g}$ added biotin $/ \mathrm{kg}$ diet. Values are means with their standard errors represented by vertical bars for eight chicks fed on hydrogenated soya-bean oil ( $\mathbf{E})$ or spent restaurant grease ().

deficiency in liver PL, elevated levels of 17:0 were found in the MO and SRG treatments, levels of $16: 1$ and $18: 3 \omega 6$ were higher in the SRG treatment, and in the HSBO treatments, $20: 4 \omega 6$ was increased while $20: 5 \omega 3$ was decreased. In heart PL, the value for $20: 4 \omega 6$ in the HSBO treatment was elevated and values for 20:3 66 were decreased in all lipid treatments due to biotin deficiency. Values for 18:0 and 18:1 were decreased for the MO and OPS treatments respectively. Levels of linoleate in liver and heart PL were highest in the MO and

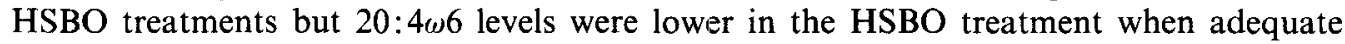
biotin was fed. Values for 20:5 3 in liver and heart PL were lowest in the MO treatment compared with the other lipids. These findings would indicate that biotin deficiency results 
in depressed elongation of $18: 3 \omega 6$ and that significant alterations in polyunsaturated fatty acids occur in liver and heart PL.

Incorporation of trans-isomers of 18:1 into the tissues of chicks receiving $400 \mu \mathrm{g}$ biotin/ $\mathrm{kg}$ was dependent on the dietary level of trans-18:1, and on tissue type and lipid fraction (Figs. 1 and 2). Much less trans-isomer was found in tissues of chicks fed on SRG than in chicks fed on HSBO. Brain tissue contained the lowest levels of trans-isomers (Fig. 1). Chicks fed on HSBO had about $16 \%$ trans-isomers in liver and slightly less in heart, lung and fat pads, but those fed on SRG had about $3 \%$ in all these tissues. There was a significantly greater incorporation of trans-18:1 into PL compared with TG in heart and liver of HSBO-fed chicks (Fig. 2). Lower levels of trans-18:1 were found in PL and TG in chicks fed on SRG.

\section{DISCUSSION}

In the present study, biotin deficiency did not result in depressed arachidonate levels. For all four dietary lipid sources, tissue levels of arachidonate were either higher or unchanged in the biotin-deficient chicks when compared with the biotin-adequate groups. In an earlier study, Watkins \& Kratzer $(1987 b)$ found arachidonate to be lower in liver total fatty acids of biotin-deficient chicks compared with their biotin-adequate counterparts at $15 \mathrm{~d}$ of age but not at $21 \mathrm{~d}$. Perhaps the changes in $20: 4 \omega 6$ levels due to biotin deficiency are agerelated or the amounts of lipids, triglycerides or phospholipids, or all three, change. Research by Gibson et al. (1984) suggests that membranes are buffered from extreme changes in fatty acid compositions which might be expected from differences in dietary lipids. Nutritional stresses may first result in reduced levels of total phospholipids rather than in altered fatty acid profiles. Unfortunately, levels of triglycerides and phospholipids could not be quantified in the present experiment.

A consistent observation in the present experiment and in previous studies with biotin-

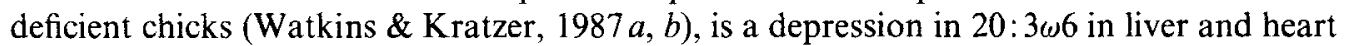
lipids. Based on fatty acid profiles of tissue lipids, it appears that elongation of $\gamma$-linoleate is reduced in biotin deficiency. Although arachidonate serves as a major precursor of prostaglandins, the prostaglandins and related compounds biosynthesized from $20: 3 \omega 6$ may be reduced in the biotin-deficient chicken. The effects of biotin deficiency on $20: 3 \omega 6$ eicosanoids has not been studied in the chicken.

The changes in linoleate levels in liver TG were particularly dramatic. In the chicks fed on MO and HSBO, there was nearly a threefold increase in $18: 2 \omega 6$ with biotin deficiency. Since the liver is the site of $90-95 \%$ of all de novo fatty acid synthesis in the chick (O'Hea \& Leveille, 1969), and a site of triglyceride synthesis, the increased levels of 18:2 $\omega 6$ may result from attenuated de novo synthesis of non-essential fatty acids. Also a block in the synthesis of dihomo- $\gamma$-linolenate due to reduced elongation of $\gamma$-linolenate during biotin deficiency may elevate linoleate (lower flux through the $\omega 6$ pathway). The latter may

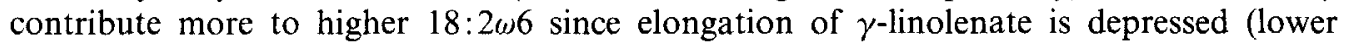
$20: 3 \omega 6$ ) in liver slices of biotin-deficient chicks (B. A. Watkins, unpublished results).

The most significant change in fatty acid profiles resulting from feeding HSBO to chicks, with adequate biotin, was a decrease in arachidonate in liver and heart PL compared with chicks fed on MO $(9 \cdot 53 v .14 .62$ and $12.76 v .15 \cdot 87)$. Levels of $\omega 3$ fatty acids $(18: 3 \omega 3$ and $20: 5(1) 3)$ in liver and heart PL of chicks fed on HSBO were increased compared with chicks fed on MO, as a consequence of the higher levels of 18:3 13 present in the HSBO. Similar trends were seen in lungs, but the effects were less dramaic (values not shown). Fatty acid profiles in brain and abdominal fat pads (not shown) were practically unchanged by feeding HSBO. These findings support previous results (Rogel \& Watkins, 1987) that arachidonate levels in liver microsomes are decreased when chicks are fed on HSBO and adequate biotin 
(200 $\mu$ g added biotin $/ \mathrm{kg}$ diet). Similar observations were also reported for rats (Lawson et al. 1983).

Positional and geometrical isomers of $18: 1$ have been shown to inhibit liver microsomal desaturation of linoleate and dihomo- $\gamma$-linolenate in vitro (Mahfouz et al. 1980, 1981). In rats, trans-18:1 isomers are more effective than cis-18:1 isomers in reducing arachidonate levels. The cis-18:1 isomers appears to compete for acylation at the 2-position of liver phosphatidylcholine, while trans-18:1 isomers are found mostly in the 1-position of liver phosphatidylcholine and phosphatidylethanolamine (Lawson et al. 1985).

In the present experiment trans-isomers were incorporated into all five tissues investigated. When HSBO (which contained $42 \%$ of $18: 1$ in the trans form) was fed, $10-16 \%$ of the tissue 18:1 found in fat pads, heart, liver and lungs was in the trans form. Tissue trans-isomer levels were much lower, less than $4 \%$, when SRG was fed (SRG contained $17 \%$ of the total $18: 1$ as trans-isomers). These results indicate that the dietary level of trans-isomers influences the incorporation of trans-18:1 into several tissue lipids except brain, which contained $1.7 \%$ trans-isomers of $18: 1$. The low level of incorporation of trans-fatty acids into brain is thought to be due to slower rates of fatty acid metabolism and turnover in this organ (Dhopeshwarkar \& Mead, 1973).

Results similar to those seen here for chicks have been reported previously for other species. Trans-isomers were incorporated into various tissues when rats were fed on hydrogenated fats (Egwim \& Sgoutas, $1971 a, b$; Emken, 1984; Blomstrand et al. 1985) and were found in human tissues studied at autopsy (Cook, 1981; Kummerow, 1983).

Values in Fig. 2 show that higher proportions of trans-18:1 accumulated in PL of heart and liver than in TG of these two organs. Studies in rats suggest that this may be due to a lowered incorporation of cis-18:1 isomers in PL (Wood, 1979). Incorporation of cis-18:1 isomers into tissues was not examined in the present study, although fatty acid profiles indicate that positional isomers were present in both cis and trans forms.

Studies with rats have shown that trans-isomers of 18:1 can exaggerate symptoms of essential fatty acid (EFA) deficiency (Privett et al. 1977; Hill et al. 1979). Unlike the rats studied by Thomassen $e t$ al. (1984), which showed elevated levels of $20: 3 \omega 9$ after 3 weeks of feeding EFA-deficient diets, the chicks in the present study fed on SRG or OPS showed no EFA-deficiency signs. Chicks do not generally develop EFA deficiency signs this early (Craig-Schmidt et al. 1986), so this was not unexpected. Feeding isomers of 18:1 at the levels chosen for the present experiment were not effective in triggering EFA deficiency symptoms at this time.

Altered linoleate metabolism may contribute to the aetiologies of FLKS and SDS. Biotin deficiency reduced the levels of dihomo- $\gamma$-linolenate in the chicken, presumably by depressing elongation of $\gamma$-linolenate (B. A. Watkins, unpublished results). On the other hand, feeding trans-18:1 fatty acids to chickens appears to lower arachidonate levels in liver. The combined effects of marginal biotin and dietary fats high in trans-fatty acids may precipitate SDS in commercial meat-type chickens reared under normal husbandry conditions. Experiments are planned to determine if practical diets containing varying levels of biotin and blended fats can influence the incidence and severity of FLKS and SDS symptoms in chickens reared to market age.

The technical assistance of Anne M. Rogel is appreciated. This research was funded by a USDA Animal Health Grant (no. 85-CRSR --2-2739). 


\section{REFERENCES}

Bannister, D. W. (1976). The biochemistry of fatty liver and kidney syndrome. Biotin-mediated restoration of hepatic gluconeogenesis in vitro and its relationship to pyruvate carboxylase activity. Biochemical Journal 156, $167-173$.

Bannister, D. W., O'Neill, I. E. \& Whitehead, C. C. (1983). The effect of biotin deficiency and dietary protein content on lipogenesis, gluconeogenesis and related enzyme activities in chick liver. British Journal of Nutrition 50, 291-302.

Bligh, E. G. \& Dyer, W. J. (1959). A rapid method of total lipid extraction and purification. Canadian Journal of Biochemistry and Physiology 37, 911-917.

Blomstrand, R., Diczfalusy, U., Sisfontes, J. \& Svensson, L. (1985). Influence of dietary partially hydrogenated vegetable and marine oils on membrane composition and function of liver microsomes and platelets in the rat. Lipids 20, 283 -295.

Cook, H. W. (1981). The influence of trans-acids on desaturation and elongation of fatty acids in developing brain. Lipids 16, 920-926.

Craig-Schmidt, M. C., Faircloth, S. A., Teer, P. A., Weete, J. D. \& Wu, C. Y. (1986). The essential fatty acid deficient chicken as a model for cystic fibrosis. American Journal of Clinical Nutrition 44, 816-824.

Dhopeshwarkar, G. A. \& Mead, J. F. (1973). Uptake and transport of fatty acids into the brain and the role of the blood-brain barrier system. Advances in Lipid Research 11, 109-142.

Dudley, P. A. \& Anderson, R. E. (1975). Separation of polyunsaturated fatty acids by argentation thin-layer chromatography. Lipids 10, 113-114.

Egwim, P. O. \& Sgoutas, D. S. $(1971 a)$. Occurrence of eicosadienoic acids in liver lipids of rats fed partially hydrogenated soybean fat. Journal of Nutrition 101, 307-314.

Egwim, P. O. \& Sgoutas, D. S. $(1971 b)$. The fatty acids of adrenal lipids from rats fed partially hydrogenated soybean fat. Journal of Nutrition 101, 315-322.

Emken, E. A. (1984). Nutrition and biochemistry of trans and positional fatty acid isomers in hydrogenated oils Annual Review of Nutrition 4, 339-376.

Gibson, R. A., McMurchie, E. J., Charnock, J. S. \& Kneebone, G. M. (1984). Homeostatic control of membrane fatty acid composition in the rat after dietary lipid treatment. Lipids 19, 942-951.

Hill, E. G., Johnson, S. B. \& Holman, R. T. (1979). Intensification of essential fatty acid deficiency in the rat by dietary trans fatty acids. Journal of Nutrition 109, 1759-1766.

Hill, E. G., Johnson, S., Lawson, L. D., Mahfouz, M. M. \& Holman, R. T. (1982). Perturbation of the metabolism of essential fatty acids by dietary partially hydrogenated vegetable oil. Proceedings of the National Academy of Sciences USA 79, 953-957.

Hood, R. L. (1977). The use of linear regression analysis in the isotope dilution assay of biotin. Analytical Biochemistry 79, 635-638.

Hood, R. L., Johnson, A. R., Fogerty, A. C. \& Pearson, J. A. (1976). Fatty liver and kidney syndrome in chicks. II. Biochemical role of biotin. Australian Journal of Biological Sciences 29, 429-441.

Hulan, H. W., Proudfoot, F. G. \& McRae, K. B. (1980). Effect of vitamins on the incidence of mortality and acute death syndrome ('flip over') in broiler chickens. Poultry Science 59, 927-931.

Kramer, T. R., Briske-Anderson, M., Johnson, S. B. \& Holman, R. T. (1984). Effects of biotin deficiency on polyunsaturated fatty acid metabolism in rats. Journal of Nutrition 114, 2047-2052

Kummerow, F. A. (1983). Modification of cell membrane composition by dietary lipids and its implications for atherosclerosis. In Biomembranes and Cell Function, pp. 29-43 [F. A. Kummerow, G. Banga, and R. P. Holmes, editors]. New York: New York Academy of Sciences

Lawson, L. D., Hill, E. G. \& Holman, R. T. (1983). Suppression of arachidonic acid in lipids of rat tissues by dietary mixed isomeric and cis and trans octadecenoates. Journal of Nutrition 113, 1827-1835.

Lawson, L. D., Hill, E. G. \& Holman, R. T. (1985). Dietary fats containing concentrates of cis or trans octadecenoates and the patterns of polyunsaturated fatty acids of liver phosphatidylcholine and phosphatidylethanolamine. Lipids 20, 262-267.

Mahfouz, M., Johnson, S. \& Holman, R. T. (1980). The effect of isomeric trans-18:1 acids on the desaturation of palmitic. linoleic and eicosa-8,1 1,14-trienoic acids by rat liver microsomes. Lipids 15, 100-107.

Mahfouz, M., Johnson, S. \& Holman, R. T. (1981). Inhibition of desaturation of palmitic, linoleic and eicosa-8,11,14-trienoic acids in vitro by isomeric cis-octadecenoic acids. Biochimica et Biophysica Acta 663, $58-68$.

Metcalfe, L. D., Schmitz, A. A. \& Pelka, J. R. (1966). Rapid preparation of fatty acid esters from lipids for gas chromatographic analysis. Analytical Chemistry 38, 514-515.

National Research Council (1984). Nutrient Requirements of Domestic Animals, Nutrient Requirements of Poultry. Washington, DC: National Academy of Sciences.

Neter, J. \& Wasserman, W. (1974). Applied Linear Statistical Models, pp. 472 482. Homewood, IL: Richard D. Irwin, Inc.

O'Hea, E. K. \& Leveille, G. A. (1969). Lipid biosynthesis and transport in the domestic chick (Gallus domesticus). Comparative Biochemistry and Physiology 30, 149-159. 
Ononiwu, J. C., Thomson, R. G., Carlson, H. C. \& Julian, R. J. (1979). Pathological studies of 'sudden death syndrome' in broiler chickens. Canadian Veterinary Journal 20, 70-73.

Privett, O. S., Phillips, F. P., Shimasaki, H., Nozawa, T. \& Nickell, E. C. (1977). Studies of effects of trans fatty acids in the diet on lipid metabolism in essential fatty acid deficient rats. American Journal of Clinical Nutrition 30, $1009-1017$.

Puddu, P., Zanetti, P., Turchetto, E. \& Marchetti, M. (1967). Aspects of liver lipid metabolism in the biotindeficient rat. Journal of Nutrition 91, 509-513.

Rogel, A. M. \& Watkins, B. A. (1987). Liver subcellular fatty acid profiles of chicks fed diets containing hydrogenated fats and varying linoleate levels. Lipids 22, 637-642.

Snedecor, G. W. \& Cochran, W. G. (1974). Statistical Methods, pp. 258-298, 343-363. Ames, Iowa: Iowa State University Press.

Steele, P., Edgar, J. \& Dondon, G. (1982). Effect of biotin supplementation on incidence of acute death syndrome in broiler chickens. Poultry Science 61, 909-913.

Thomassen, M. S., Rortveit, T., Christiansen, E. N. \& Norum, K. R. (1984). Change in the content of $n-6$ fatty acids in liver phospholipids in rats as a consequence of partially hydrogenated dietary oils. British Journal of Nutrition 51, 315-322.

Watkins, B. A. \& Kratzer, F. H. (1987a). Tissue lipid fatty acid composition of biotin-adequate and biotindeficient chicks. Poultry Science 66, 306-313.

Watkins, B. A. \& Kratzer, F. H. (1987 b). Dietary biotin effects on polyunsaturated fatty acids in chick tissue lipids and prostaglandin $\mathrm{E}_{2}$ in freeze-clamped hearts. Poultry Science 66, 1818-1828.

Wood, R. (1979). Incorporation of dietary cis and trans octadecenoate isomers in the lipid classes of various rat tissues. Lipids 14, 972-982. 